\title{
Study on the surface modification of bamboo pulp fibers through AGET-ATRP grafting
}

\author{
Jianfei $\mathrm{LI}^{1, a}$, Xiumei ZHANG ${ }^{2} \mathrm{~b}^{*}$, Jian $\mathrm{WANG}^{3, \mathrm{c}}$ \\ ${ }^{1}$ College of Materials and Textiles, Zhejiang Sci-Tech University, Hangzhou, 310018, China \\ ${ }^{2}$ College of Materials and Textiles, Zhejiang Sci-Tech University, Hangzhou, 310018, China \\ ${ }^{3}$ College of Materials and Textiles, Zhejiang Sci-Tech University, Hangzhou, 310018, China \\ aemail: fei890116@163.com, ${ }^{\text {b*e}}$ email: xiumei@zstu.edu.cn, \\ cemail: xiaobing0121@163.com
}

Keywords: bamboo pulp fibers (BPF); initiator; graft; modification; AGET-ATRP

\begin{abstract}
In order to improve the surface compatibility of bamboo pulp fibers (BPF) with plastic materials, enlarge the application fields, BPF macromolecule which was modified by 2-bromoisobutyryl bromide (BIBB) was chosen as the initiator. Copper(II)bromide/ $N, N, N^{\prime}, N^{\prime}, N^{\prime}$ pentamethyldiethylenetriamine (PMDETA) was used as the catalyst and ascorbic acid was selected as the reducing agent. Surface grafting modification of BPF with monomer isoprene was prepared by the method of Activators Regenerated by Electron Transfer Atom Transfer Radical Polymerization (AGET-ATRP), meanwhile the effects of parameters such as monomer content, catalyst content, response time and temperature on modification were determined. The results showed that the significant changes of the surface chemical groups in FTIR spectrogram demonstrated the occurrence of grafting reaction and monomer isoprene was successfully grafted onto the surface of BPF. And the changes in XRD spectrogram showed the changes of crystal structure, which verified the veracity of the results.
\end{abstract}

\section{Introduction}

Bamboo is a kind of common plant and is dispersed all over the world. It has been reported that its specific strength is much higher than that of other materials (mild steel, polyester resin, glass-reinforced plastic with woven rovings and chopped strand mat) [1]. It may be said that BPF can compete with carbon fiber, glass fiber and polymer fiber as reinforcement filler thanks to its abundance, cheap and renewable resource [2]. Cellulose-based materials are, therefore, of great interest in many applications, especially when considering the increasing environmental issues. For some applications, however, BPF surface is highly hydrophilic due to a large number of hydroxyl groups on the molecular structure, which cause the poor compatibility between BPF and some polymers (PP, PE, PS, PVC, etc.). This is often essential to achieve better adhesion when using fibers as reinforcement in composites, especially since the matrix often is a hydrophobic polymer [3].

Grafting of cellulose via Atom Transfer Radical Polymerization (ATRP) is a mild approach that does not need any pretreatment with base. It is one of the most successful "controlled/living" radical polymerization (CRP) developed during recent years [4][5][6]. However, ATRP has some limitations. Since ATRP is initiated by a redox reaction between an initiator with a radically transferable atom or group and a catalyst complex comprising a transition metal compound in a lower oxidation state, the transition metal complexes can be easily oxidized to a higher oxidation state. Therefore, to obtain consistent results, special handling procedures are required, and the preformed catalysts must be stored under an inert atmosphere [7]. Oxygen or other oxidants should be removed from the system prior to addition of the catalyst in the lower oxidation state; therefore, the process of catalyst complex handling can be challenging [8][9]. This paper describes a new method for formation of an active catalyst, a procedure for preparing an "activator generated by electron transfer" for ATRP (AGET-ATRP), which overcomes those prior problems by using an electron transfer rather than organic radicals to reduce the higher oxidation state transition metal 
[10].

In this study, in order to improve the surface compatibility of bamboo pulp fibers (BPF), a planning was made to graft some groups onto the bamboo pulp fibers by the method of AGET-ATRP [11], and the grafted product was investigated by FTIR, XRD.

\section{Materials and Methods}

\section{Experimental Materials}

Bamboo pulp fibers (BPF obtained from Zhejiang) were used as the cellulose material. Ethanol acetone (AR, Zhanwang), tetrahydrofuran (THF, AR, Yongda) and triethylamine (TEA,AR,Yongda), 4-(dimethylamino)pyridine(DMAP,99\%, aladdin), 2-bromoisobutyryl-bromide(BIBB,98\%,MACKLI $\mathrm{N}$ ), N,N-dimethylformamide(DMF,AR, GaojingFineChemical), $N, N, N^{\prime}, N^{\prime}, N^{\prime}$-pentamethyldiethylenetr iamine(PMDETA,98\%,aladdin), copper(II)bromide(Cu(II)Br2,99\%, aladdin), ascorbic acid(VC, AR, Kermel), isoprene( $99 \%$, aladdin) were purchased from Hangzhou Mike Chemical Reagent Co. Ltd. Preparation of bamboo pulp fibers macro-initiators (BPF-Br)

The dry bamboo pulp fibers (BPF, $0.5 \mathrm{~g}$ ) were taken into the dissociation reactor to disperse, then rinsed the dissociated BPF with acetone and THF, they were stored after vacuum filtration, the purpose of rinsing is removing impurities. Because water has an effect on the next esterification reaction, the purpose of previous operation is removing the water. The three-necked round bottom flask $(100 \mathrm{ml})$ was placed in a constant temperature water bath, DMAP $(0.5 \mathrm{~g})$ was added into flask, then THF $(50 \mathrm{ml})$ and TEA $(1.5 \mathrm{ml})$ were taken into the flask to dissolve DMAP. The dissociated BPF was tore into three-necked flask, and mixed with the rotor. After mixing, as the flask was cooled down to $0{ }^{\circ} \mathrm{C}$ with an ice-bath, BIBB $(1 \mathrm{ml})$ was slowly added into the flask droplet by droplet using a hypodermic syringe. The reaction conditions must be closed. After the mixture was added, the flask was heated in a water-bath at $60{ }^{\circ} \mathrm{C}$ and held for $24 \mathrm{~h}$ under $\mathrm{N}_{2}$ atmosphere. Finally, the precipitate was dried at $60{ }^{\circ} \mathrm{C}$ in a vacuum oven for $24 \mathrm{~h}$; thus, the BPF-Br was prepared. The process is outlined in Figure 1.

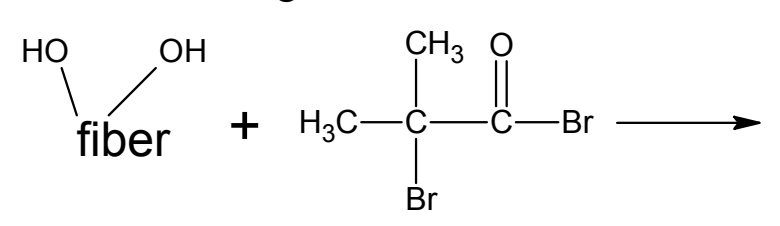<smiles></smiles>

Fig.1. Preparation of the macro-initiator agent

\section{Grafting Isoprene to the BPF-Br surface}

DMF $(10 \mathrm{ml})$ was added into a $100 \mathrm{ml}$ three-neck round bottom flask, and then add $\mathrm{CuBr}_{2}(0.067$ $\mathrm{g}), \operatorname{PMDETA}(0.115 \mathrm{ml}), \mathrm{VC}(0.1585 \mathrm{~g})$ in the flask. Isoprene $(6.8 \mathrm{~g})$ and $\mathrm{BPF}-\mathrm{Br}$ were taken into the flask after stirring. The reaction temperature is $30^{\circ} \mathrm{C}$ and the reaction time is $2 \mathrm{~h}$. The grafted sample was thoroughly rinsed with ethanol, acetone and THF [12], then followed by Soxhlet extraction for another $1 \mathrm{~h}$ to completely remove any unreacted and unbound materials. Finally, they were dried under vacuum conditions to constant weight at $60{ }^{\circ} \mathrm{C}$. The process is outlined in Figure 2.

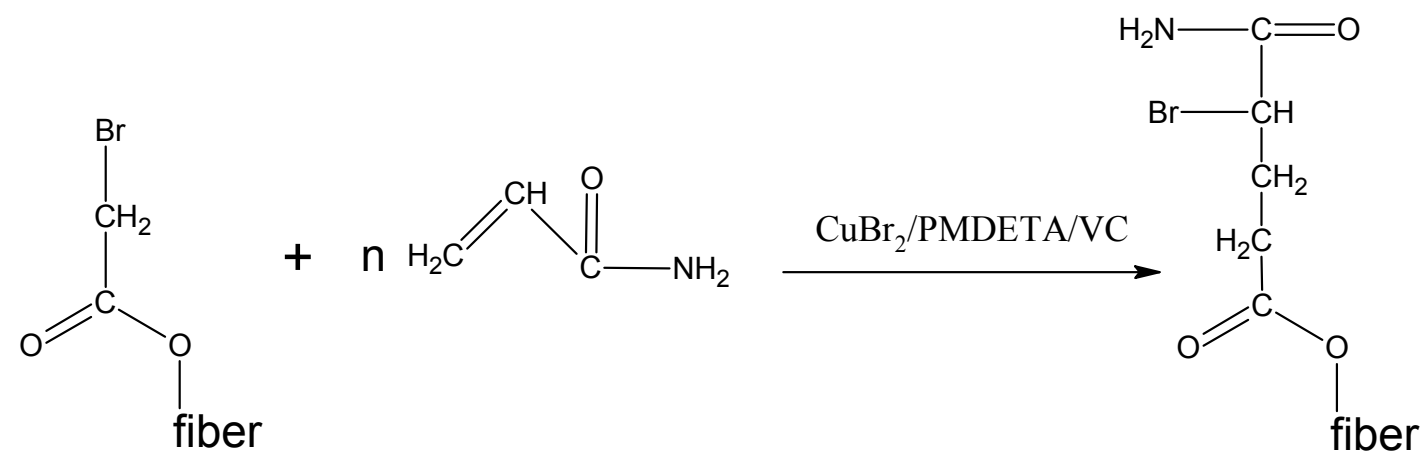

Fig.2. The AEGT-ATRP graft polymerization of isoprene 


\section{The impact of parameter}

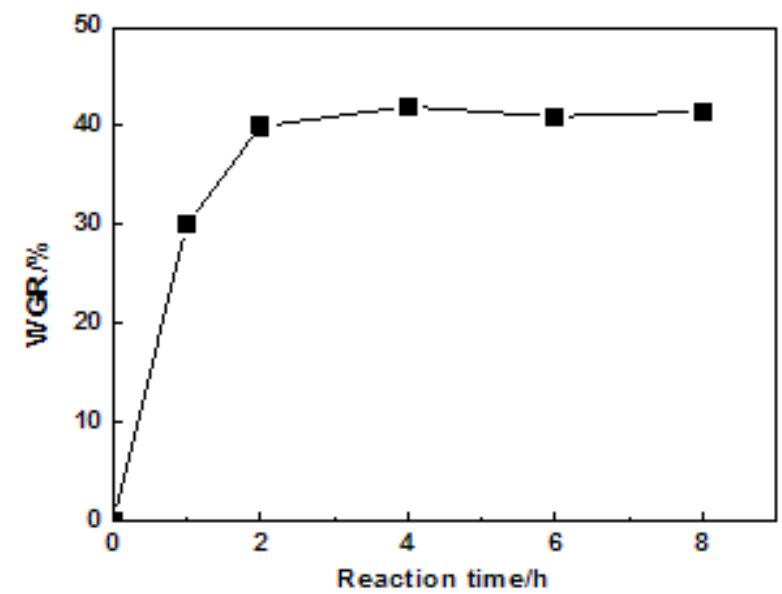

Fig.3. Effect of monomer consumption on reaction product

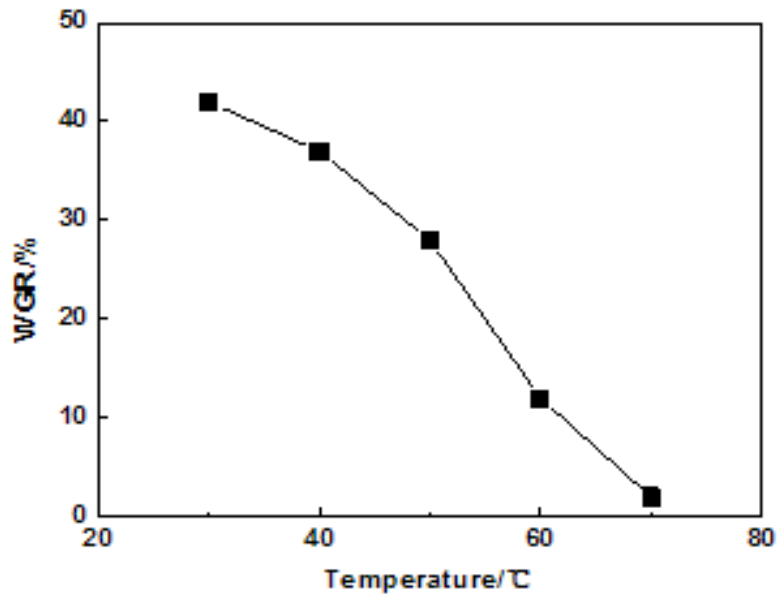

Fig.4. Effect of catalyst consumption on reaction product

\section{Monomer \& catalyst content}

If other conditions remained unchanged, the amount of isoprene monomer was changed to observe and analyze the growth rate of product. And the result is depicted in Figure 3 . With the amount of isoprene monomer increasing, the product growth rate was increasing constantly. Therefore, the amount of reactive monomer is determined as $5 \mathrm{~mol} \cdot \mathrm{L}^{-1}$ finally.

If other conditions remained unchanged, the amount of catalyst was changed to observe and analyze the growth rate of product. And the result is depicted in Figure 4. With the amount of catalyst increasing to $15 \mathrm{mmol} \cdot \mathrm{L}^{-1}$, the product growth rate was increasing, then decreasing. Therefore, the amount of reactive catalyst is determined as $15 \mathrm{mmol} \cdot \mathrm{L}^{-1}$ finally.

Reaction time \& temperature

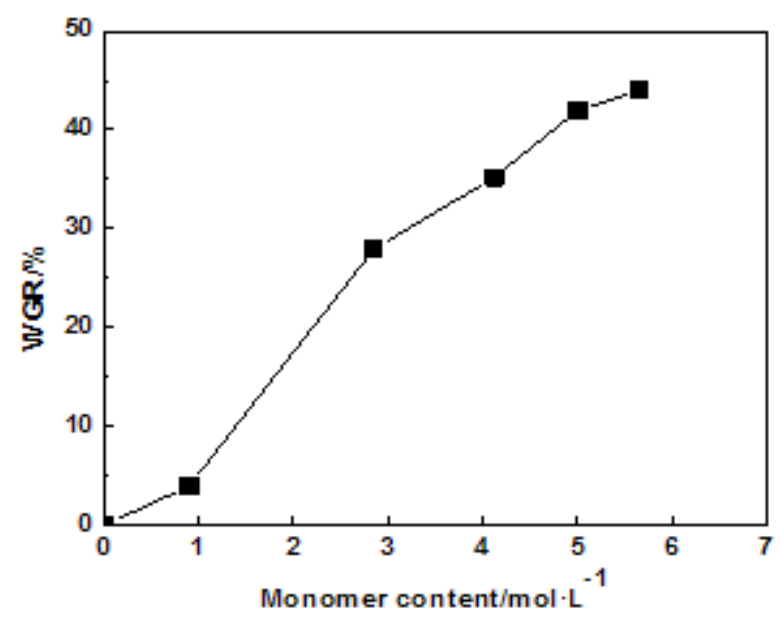

Fig.5. Effect of reaction time on the reaction product

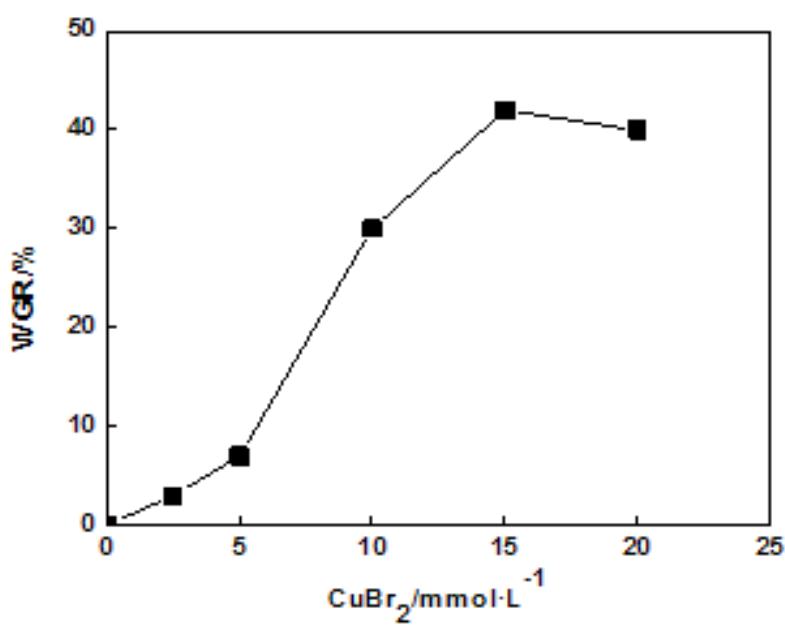

Fig.6. Effect of reaction temperature on the reaction product

If other conditions remained unchanged, the reaction time was changed to observe and analyze the growth rate of product. And the result is depicted in Figure 5. Within 1h, the reaction product growth rate continued to increase, the product growth rate tended to become smaller between $1 \mathrm{~h}$ and $2 \mathrm{~h}$, and the product growth rate was unchanged after $2 \mathrm{~h}$. Therefore, the reaction time is determined as $2 \mathrm{~h}$ finally.

If other conditions remained unchanged, the reaction temperature was changed to observe and 
analyze the growth rate of product. And the result is depicted in Figure 6. The reaction temperature was changed from $30{ }^{\circ} \mathrm{C}$ to $70{ }^{\circ} \mathrm{C}$ gradually, the weight of products continued to decrease. The reason is that the boiling point of isoprene is $34{ }^{\circ} \mathrm{C}$, high temperature caused isoprene into a gas, which was not easy to react with macro-initiators. Therefore, the reaction temperature is determined as $30{ }^{\circ} \mathrm{C}$ finally.

\section{Results and Analysis}

\section{The analysis of FTIR}

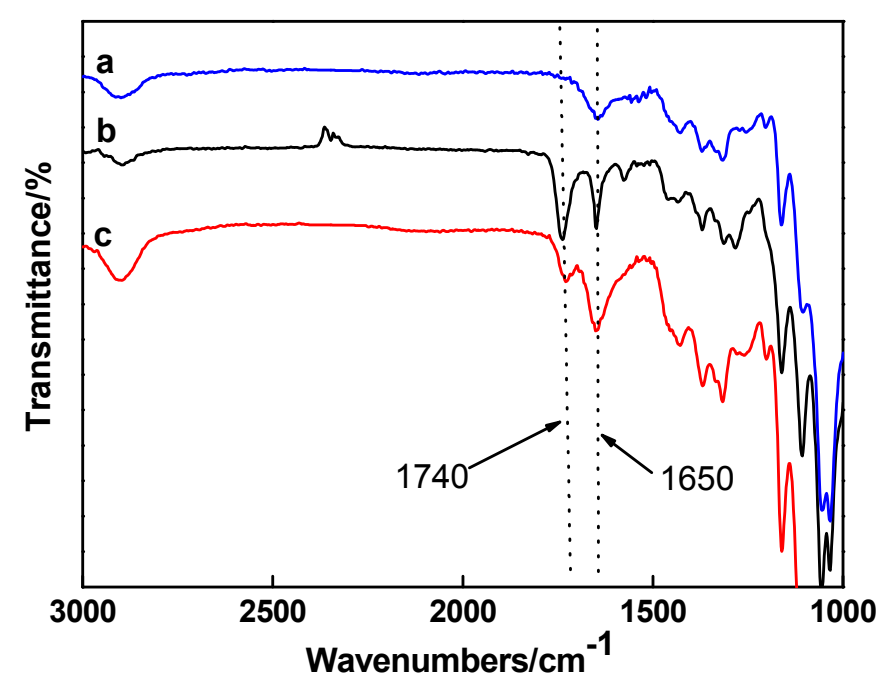

Fig.7. FTIR spectra of (a) pristine BPF, (b) purified BPF-Br and (c) BPF-g-PI

The chemical composition of the BPF surface was examined by Fourier transform infrared (FTIR) spectroscopy (Nicolet 5700, Thermo scientific, American). Test results are depicted in Figure 7. As can be seen, after the reaction, one new peak was observed at $1740 \mathrm{~cm}^{-1}$, this is an unsaturated ester $\mathrm{C}=\mathrm{O}$ stretching vibration absorption peak. At $1650 \mathrm{~cm}^{-1}$, that is $\mathrm{C}=\mathrm{C}$ double bond stretching vibration peak. Compared with (b), the ester contraction peak of (c) becomes weaken at $1740 \mathrm{~cm}^{-1}$, but the peak of (c) is wider than (a), (b) at $1740 \mathrm{~cm}^{-1}$, and stretching is more intense. This suggests that isoprene has been grafted onto the fibers successfully.

The analysis of XRD

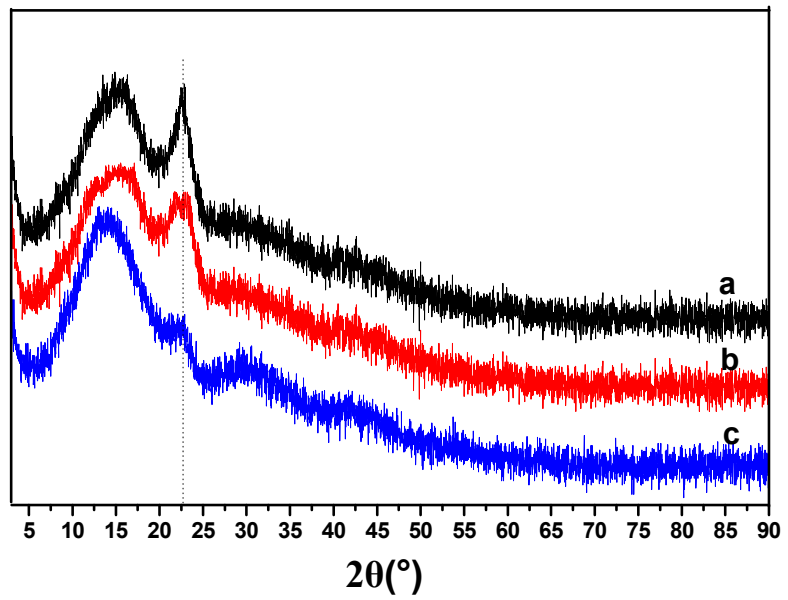

Fig.8. XRD pattern of (a) pristine BPF, (b) purified BPF-Br and (c) BPF-g-PI

To prove that BPF is grafted successfully, the study also carried out X-ray diffraction (XRD) analysis (ARL XTRA, Thermo ARL, Switzerland) before and after the modification of BPF. Test results are depicted in Figure 8. Pristine BPF (a) has strong diffraction peaks at near $2 \theta=23.5^{\circ}$, 
this is crystal diffraction peak of bamboo pulp fibers (102). In contrast, the position of the BPF-Br (b) crystallization peak did not change significantly, but the intensity of the diffraction peak has changed significantly. The crystallinity of untreated BPF is $54.3 \%$, while the crystallinity of BPF-Br is $27.6 \%$, This shows that the crystallinity of BPF grafted initiator is decreased. This is because the hydroxyl groups of BPF surface reacted with BIBB, the cellulose crystalline regions of BPF was damaged. Original part of the BPF diffraction peaks almost disappear after grafting isoprene. This is due to the hydroxy of cellulose was reduced after grafting. And the introduction of isoprene molecular chains changed the original aggregation structure of BPF, and hindered the function of association among cellulose molecules. The breakage of crystalline regions resulted in decreasing of crystallinity. As time was prolonged, the cellulose crystalline regions were more damaged fully [13]. In contrast with (a) and (b), diffraction peak intensity of (c) is decline or even disappear at $2 \theta$ $=23.5^{\circ}$. This suggests that isoprene has been grafted onto the fibers successfully.

\section{Conclusion}

Ultimately, this study determined the optimal experimental parameters by controlling these types of reaction conditions (the amount of the monomer, the catalyst, reaction time and temperature). In conclusion, isoprene has been grafted onto the surface of the BPF by using the method of AGET-ATRP. The modified BPF was characterized using FTIR, XRD in detail. By analyzing the grafted BPF with FTIR and XRD, it could be concluded that isoprene has been grafted onto the BPF surface successfully.

\section{Acknowledgement}

In this paper, the research was sponsored by the Science and Technology Program of Zhejiang Province of China (Project No. 2013C32085).

\section{References}

[1] Lakkad S C, Patel J M. Mechanical properties of bamboo, a natural composite [J]. Fibre Science \& Technology, 1981, 14(4):319-322.

[2] Klemm D, Heublein B, Fink H, et al. Cellulose: Fascinating Biopolymer and Sustainable Raw Material [J]. Cheminform, 2005, 36(36):3358-3393.

[3] S H, E O, A C, et al. ARGET ATRP for Versatile Grafting of Cellulose Using Various Monomers [J]. Acs Applied Materials \& Interfaces, 2009, 1(11):2651-2659.

[4] Matyjaszewski K, Gaynor S, Greszta D, et al. 'Living' and controlled radical polymerization [J]. Journal of Physical Organic Chemistry, 1995, 8(4):306-315.

[5] MATYJASZEWSKI K. Controlled/Living Radical Polymerization, Progress in ATRP, NMP, and RAFT [J]. American Chemical Society, 2000, 768(12):4167-4171.

[6]Gao H, Matyjaszewski K, Gao H, et al. Synthesis of Molecular Brushes by "Grafting onto" Method: Combination of ATRP and Click Reactions[J]. Journal of the American Chemical Society, 2007, 129(20):6633-9.

[7] Jakubowski W, Matyjaszewski K. Activator Generated by Electron Transfer for Atom Transfer Radical Polymerization [J]. Macromolecules, 2005, 38(10):4139-4146.

[8] Matyjaszewski K. Mechanistic features and radical intermediates in atom transfer radical polymerization [J]. Macromolecular Symposia, 2002, (1):71-82.

[9] A C, EE. M. ATRP grafting from cellulose fibers to create block-copolymer grafts.[J]. Biomacromolecules, 2003, 4(6):1740-1745. 
[10] Xingbing Y, Liwu Z, Jing L, et al. Research Progress of Activator Generated by Electron Transfer for Atom Transfer Radical Polymerization[J]. Paint\&Coatings Industry, 2010.

[11] Min K, Gao H, Matyjaszewski K. Preparation of homopolymers and block copolymers in miniemulsion by ATRP using activators generated by electron transfer (AGET).[J]. Journal of the American Chemical Society, 2005, 127(11):3825-30.

[12] Liu Z, Sun C, Liu Z, et al. Adjustable wettability of methyl methacrylate modified ramie fiber [J]. Journal of Applied Polymer Science, 2008, 109(5):2888-2894.

[13] Yu F, Yang W, Song J, et al. Investigation on hydrophobic modification of bamboo flour surface by means of atom transfer radical polymerization method [J]. Wood Sciences \& technology, 2014, 48(2):289-299. 